\title{
Imperfect imitation can enhance cooperation
}

\author{
C. P. Roca ${ }^{1(a)}$, J. A. Cuesta ${ }^{1}$ and A. SÁnchez ${ }^{1,2,3}$ \\ ${ }^{1}$ GISC/Departamento de Matemáticas, Universidad Carlos III de Madrid - Madrid, Spain, EU \\ ${ }^{2}$ Instituto de Biocomputación y Física de Sistemas Complejos (BIFI), Universidad de Zaragoza - Zaragoza, \\ Spain, EU \\ ${ }^{3}$ Instituto de Ciencias Matemáticas CSIC-UAM-UC3M-UCM - Madrid, Spain, EU
}

received 1 June 2009; accepted in final form 6 August 2009

published online 7 September 2009

PACS 89.65.-s - Social and economic systems

PACS 02.50.Le - Decision theory and game theory

PACS 87.23. Kg - Dynamics of evolution

\begin{abstract}
The promotion of cooperation on spatial lattices is an important issue in evolutionary game theory. This effect clearly depends on the update rule: it diminishes with stochastic imitative rules whereas it increases with unconditional imitation. To study the transition between both regimes, we propose a new evolutionary rule, which stochastically combines unconditional imitation with another imitative rule. We find that, surprisingly, in many social dilemmas this rule yields higher cooperative levels than any of the two original ones. This nontrivial effect occurs because the basic rules induce a separation of timescales in the microscopic processes at cluster interfaces. The result is robust in the space of $2 \times 2$ symmetric games, on regular lattices and on scale-free networks.
\end{abstract}

Copyright (C) EPLA, 2009

Why individuals cooperate is a key problem in a wide range of disciplines [1], being studied theoretically mainly within the framework of evolutionary game theory [2]. One of the proposed mechanisms to explain cooperation is network reciprocity [3], and so different population structures are known to have an influence on the evolutionary outcome of social dilemmas [4]. All these models incorporate some kind of evolutionary dynamics [5], whose update rules may play a crucial role in the results. For example, the well-known promotion of cooperation in Prisoner's Dilemma enforced by spatial lattices [6] is linked to a particular non-stochastic rule (unconditional imitation), and this effect is greatly reduced if another dynamics, imitative but stochastic, is employed $[7,8]$.

Imitation is a well-known feature of human behavior $[9,10]$. By an imitative dynamics we understand an update rule that makes individuals copy, within certain constraints, the strategy of those other players that are doing better, or, in game theoretical terms, that are obtaining higher payoffs from the game. In the case of network reciprocity, the range of individuals that every player takes into account is limited to her nearest neighbors on the network. Two of the most frequently used imitative dynamics in the literature are the unconditional imitation rule and the replicator rule [4]. In the former,

\footnotetext{
${ }^{(a)}$ E-mail: cproca@math.uc3m.es
}

individuals acquire the strategy of the player with the maximum payoff in their neighborhood including themselves [6]. In the latter, players choose a neighbor at random and copy her strategy with probability proportional to the difference of payoffs, provided the neighbor's payoff is greater than hers. It can be proven [11] that in large well-mixed populations this last rule induces an evolutionary dynamics equal to the replicator equation, thus leading the population to asymptotic states very closely related to the evolutionary stable equilibria of the game [5]. In structured populations, though, the evolutionary outcome may greatly differ from the equilibria of the game [4], and different evolutionary dynamics can yield very different results, as in the example above. Therefore, it is very relevant to study the dependence of the promotion of cooperation on the evolutionary rules and their robustness against perturbations.

In this work, we focus on this issue within the framework of unconditional imitation and spatial lattices. It may be argued that sometimes individuals can be able to identify the strategy of all their neighbors and correctly assess their earnings, but it is difficult to assume that all this complex process may proceed without errors or disturbances. Indeed, previous work has pursued this enquiry, using a Moran-like rule with weighted probabilities [12], finding a progressive lowering of the cooperation levels as the rule differs from unconditional imitation. Here 
we use a different approach, which consists in stochastically combining unconditional imitation with another less demanding imitative rule. Thus, when an individual is to update her strategy, she follows unconditional imitation with probability $1-\rho$ and the other rule with probability $\rho$ (the other rule is the replicator rule, unless stated otherwise). The resulting evolutionary rule, which we call the $\rho$-rule in the following, is local and imitative, and the parameter $\rho \in[0,1]$ measures the perturbation introduced. Notice that this setting differs from a mutation scheme, as the players do not acquire indefinitely, or until the next mutation, the secondary rule.

We have studied computationally the outcome of the $\rho$-rule with $2 \times 2$ symmetric games, which are games with 2 players who choose between 2 strategies, with no difference in role. We use the following parametrization of the payoff matrix $[7,8,13]$ :

$$
\begin{aligned}
& \text { C D } \\
& \mathrm{C} \quad\left(\begin{array}{cc}
1 & S \\
T & 0
\end{array}\right) \text {, }
\end{aligned}
$$

where rows represent the strategy ( $\mathrm{C}$ for cooperate and $\mathrm{D}$ for defect) of the player who obtains the payoff and columns that of her opponent. Restricting parameters to the square $-1<S<1,0<T<2$, we have the Harmony game [14] $(0<S, T<1)$ and three classic social dilemmas: the Prisoner's Dilemma [15] $(-1<S<0,1<T<2)$, the Stag Hunt game [16] $(-1<S<0<T<1)$, and the HawkDove [17] or Snowdrift game [18] $(0<S<1<T<2)$. Therefore each game corresponds to a unit square in the $S T$-plane. We have considered square lattices with 4- and 8-neighborhoods, doing the update synchronously (all players play and then they simultaneously update their strategy) or asynchronously (players play and update their strategy sequentially in random order).

Figure 1 displays an example of the nontrivial behavior obtained with the $\rho$-rule, showing the asymptotic fraction of cooperators $x^{*}$ as a function of the probability $\rho$, for a Prisoner's Dilemma of parameters $S=-0.7$ and $T=1.1$. With this game, both $\rho=0$ (unconditional imitation) and $\rho=1$ (replicator rule) result in full defection, but a large range of values of $\rho$ yield almost full cooperation. This phenomenon resembles resonance-like behavior found in other game-theoretical models $[19,20]$, albeit in this case the effect is very large and occurs for a wide range of $\rho$.

The explanation of this counter-intuitive result lies in the different microscopic dynamical processes, with greatly differing timescales, that the update rules induce. Their combination gives rise to a ratchet effect, something that can yield paradoxical outcomes, as in the famous Parrondo's games [21-23]. On regular lattices both rules enhance cooperation by means of the formation and growth of clusters of cooperators [6]. With unconditional imitation clusters grow rapidly and mostly with flat interfaces, whereas with the replicator rule clusters grow much more slowly and have much rougher interfaces [7].

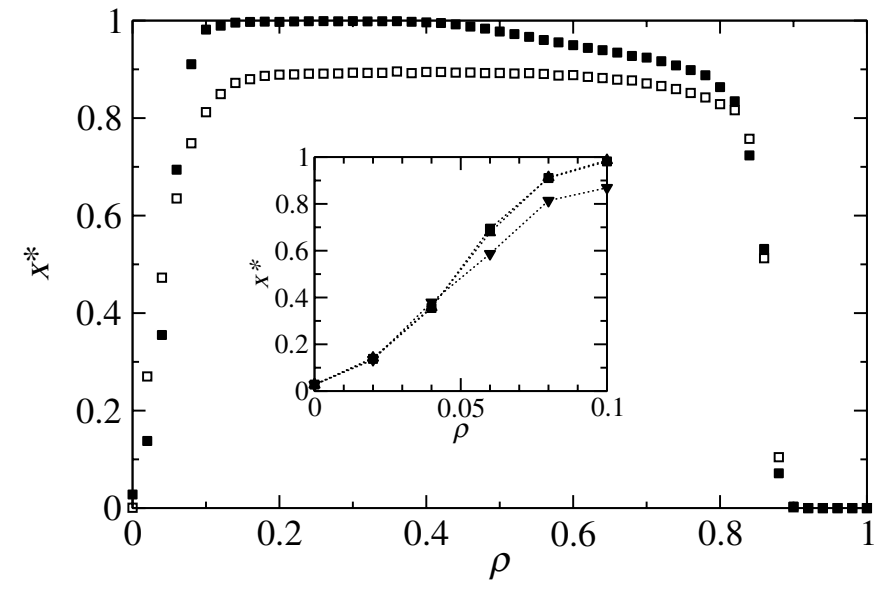

Fig. 1: Asymptotic density of cooperators $x^{*}$ in the Prisoner's Dilemma $(S=-0.7, T=1.1)$, as a function of $\rho$, with synchronous (filled squares) or asynchronous (empty squares) update. Population size $N=10^{4}$ individuals, arranged on a square lattice with 8 neighbors and periodic boundary conditions. The initial fraction of cooperators is 0.5 , randomly distributed. Simulation time is $10^{4}$ generations. The asymptotic values are obtained as the mean over the last $10^{3}$ generations, averaged over 100 realizations. Inset: results with population sizes $N=2500$ (triangles down), $N=10^{4}$ (squares) and $N=4 \times 10^{4}$ (triangles up). The results for $N=10^{4}$ and $N=4 \times 10^{4}$ are virtually identical, and all three are very similar for $\rho>0.2$. Lines are a guide to the eye.

Let us consider a flat interface of opposing cooperators and defectors in a 8-neighbor lattice, as depicted in fig. 2(a). With unconditional imitation $(\rho=0)$ defectors at the boundary become cooperators if $5+3 S>3 T$. Otherwise the interface remains frozen, and clusters of cooperators are not able to grow. With the replicator rule $(\rho=1)$, cooperators at the boundary become, with a certain probability, defectors and the interface roughens progressively. When $\rho>0$ the flat interface slowly becomes rougher because of those players that happen to follow the replicator rule, and then nearby defectors that happen to follow unconditional imitation rapidly become cooperators precisely because of the irregularities at the interface. For example, starting from the flat interface in fig. 2(a) a cooperator will use the replicator rule and compare payoff with one of the opposing defectors with probability $3 \rho / 8$, becoming with some probability a defector (fig. 2(b)). If this player follows unconditional imitation in the next update she will switch back to cooperation. If not, with a certain probability, one of the nearby defectors becomes herself a cooperator, using again the replicator rule, thus producing the kink of fig. 2(c). This configuration is critical because if nearby defectors follow unconditional imitation in the next time steps, they will immediately become cooperators, and the interface will advance one step to the right (figs. 2(d), (e)). Note the asymmetry that induces this ratchet effect: unconditional imitation makes defectors at interface irregularities become cooperators 


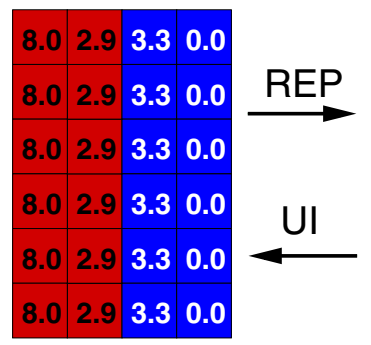

(a)

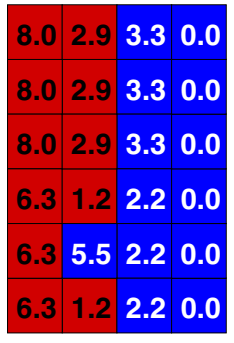

(b)
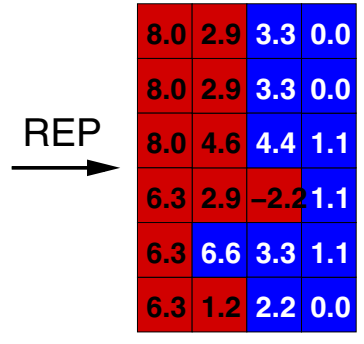

(c)
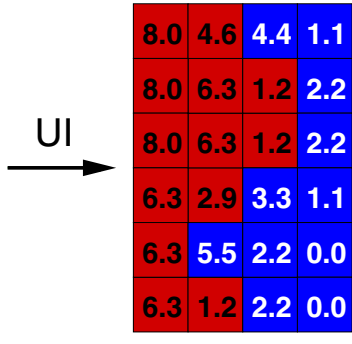

(d)

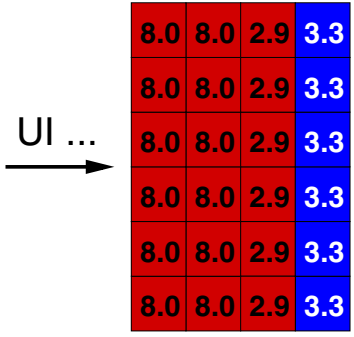

(e)

Fig. 2: (Colour on-line) Flat interface evolution, with synchronous update, in the Prisoner's Dilemma of fig. 1 and $\rho>0$. Each position shows the strategy and payoff of a player. Cooperators are depicted in red and defectors in blue. Changes of strategy made under unconditional imitation are labeled with UI, and those under the replicator rule with REP.
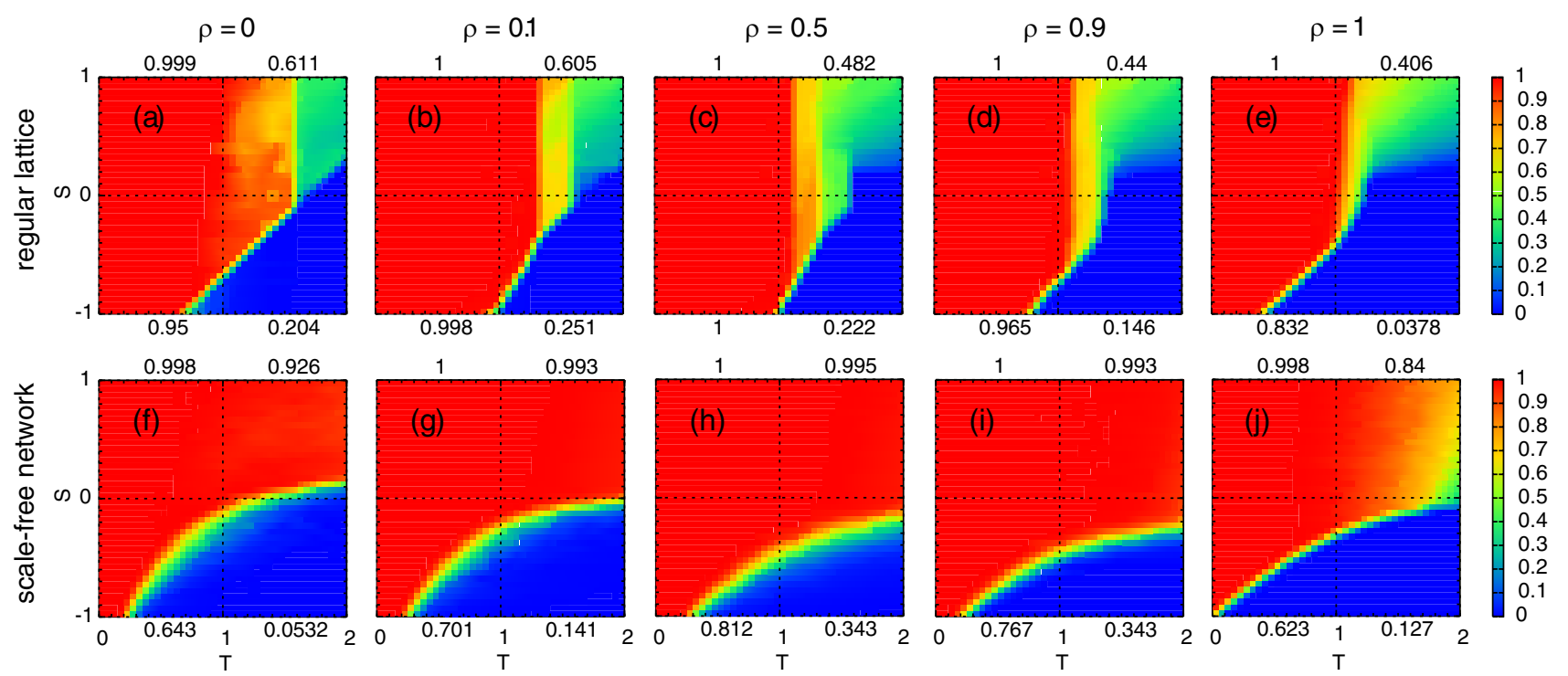

Fig. 3: (Colour on-line) Asymptotic fraction of cooperators, for different social dilemmas defined by $S$ and $T$, as $\rho$ varies from 0 to 1 . The population is arranged on a square lattice (first row, panels (a) to (e)) or a Barabási-Albert scale-free network (second row, panels (f) to (j)), both of mean degree equal to 8. The update is synchronous. Remaining parameters are as in fig. 1. Notice the numbers above Harmony and Snowdrift (below Stag Hunt and Prisoner's Dilemma) squares, which show the average asymptotic cooperation achieved in each game square (see text).

but not vice versa, producing an irreversible movement of the cluster interface. Simulations show this kind of growth process, where irregularities at interfaces of clusters appear in configurations similar to that of fig. 2 and also in cluster corners, giving rise to cascades of conversions from defectors to cooperators along the interfaces.

As is typical for dynamics driven by unconditional imitation $[7,8]$, the population ends up in full cooperation as long as there are, at initial time, small clusters of cooperators that resist defector invasion and grow. Greater population sizes mean larger probabilities of these clusters to occur, and so there is a dependence on system size in the region close to $\rho=0$ (inset of fig. 1). Note also that the time of convergence diverges in the limit $\rho \rightarrow 0$, because the interface instabilities explained above take place with a probability proportional to $\rho^{2}$, as they require two players at the interface to follow (with probability $\rho$ ) the replicator rule.

The fact that this mechanism is rooted in the basic microscopic processes that the update rules cause is a hint that it should occur with other games where these processes are also known to take place $[7,8]$. Figures 3(a)-(e) illustrate this point, displaying the asymptotic fraction of cooperators in the same network topology as fig. 1, for different values of $\rho$ and with the games introduced above: Harmony (upper left square), Stag Hunt (lower left), Snowdrift (upper right) and Prisoner's Dilemma (lower right). To have a measure of the global influence on each kind of game, we have calculated the average value of the asymptotic fraction of cooperators achieved in each game square $[7,8]$. The resulting numbers are displayed beside the corresponding square (above for 
Harmony and Snowdrift games, below for Stag Hunt and Prisoner's Dilemmas). Notice how the transition between full cooperation and full defection of fig. 3(a) advances in the Prisoner's Dilemma square for intermediate values of $\rho$. With unconditional imitation $(\rho=0)$ this boundary is given by the payoff equality between cooperators and defectors at both sides of a flat interface [6], which for the setting of fig. 3 (a) corresponds to $T-S=5 / 3$. With $0<\rho \ll 1$ (figs. 3(b), (c)), this boundary is instead given by $2 T-S=3$, which is precisely the condition for the payoff equality of the players who determine the start of cascades at the interfaces, namely the players on the third row, second and third columns, of fig. 2(c). Note also that the transition line at $T=8 / 5$, which determines the instability of inwards corners of cooperators [6,24], is preserved for $\rho>0$, and that a new line appears at $T=4 / 3$, which gives the condition for which the irregularities of cluster interfaces, caused by the replicator rule, trigger a defector invasion under unconditional imitation (compare the payoff of the defector on the fifth row and second column of fig. 2(c) with that of the cooperator two positions to the left).

Considering other stochastic imitative rules to be used in the $\rho$-rule, like the multiple replicator or the Moran rules $[4,7]$, does not significantly change the results. In fact, any imitative local rule that destabilizes the interfaces of clusters of cooperators and that works on a slower timescale than unconditional imitation (as it is expected for stochastic rules) will produce qualitatively similar results. We have found this phenomenon even with a random local rule (players just adopt the strategy of one randomly chosen neighbor), providing that $\rho \ll 1$. The effect is also robust against changes in the degree of the network and is found with synchronous and asynchronous update.

Another important variation to consider in this model is that of the topology of the underlying network. Apart from the clustering of spatial lattices [6], the degree heterogeneity of scale-free networks is another topological property known to have an important impact on the evolution of cooperation [8,13]. In consequence, we have also studied the evolutionary outcome that the $\rho$-rule yields on populations structured according to BarabásiAlbert scale-free networks [25]. The results are presented in figs. 3(f)-(j).

From the point of view of the evolution of cooperation fig. 3 shows that, for a large range of $\rho$, practically full cooperation is obtained in Stag Hunt (Snowdrift) games on spatial (scale-free) networks. Very importantly, with the $\rho$-rule the population achieves full cooperation precisely in those games where the underlying population structure has its greatest impact [8]. Regarding the Prisoner's Dilemma, we have studied the average asymptotic cooperation $\left\langle x^{*}\right\rangle_{\mathrm{PD}}$ in the corresponding game square, obtaining the results shown in fig. 4, both for spatial lattices (a) and scale-free networks (b). Interestingly, some differences appear depending on the synchronicity of the update.

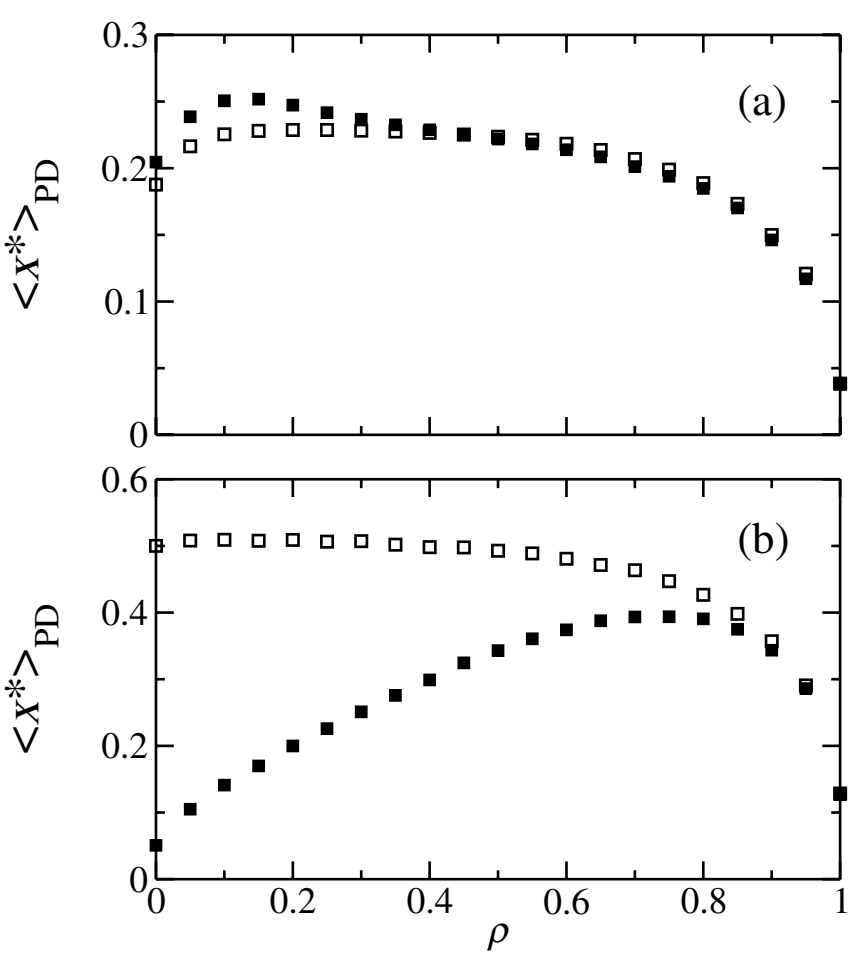

Fig. 4: Average asymptotic cooperation in the Prisoner's Dilemma $\left\langle x^{*}\right\rangle_{\mathrm{PD}}$, as a function of $\rho$. Populations are distributed on square lattices (a) or scale-free networks (b), both of mean degree $=8$. The update is synchronous (filled squares) or asynchronous (empty squares). Remaining parameters are as in fig. 1. Notice the different scales of $\left\langle x^{*}\right\rangle_{P D}$ in (a) and (b).

With synchronous update, a maximum average cooperation is achieved for an optimum $\rho$, whereas for asynchronous update the result is rather a plateau over a large range of $\rho$. On spatial lattices the differences are small, but on scale-free networks they are strikingly large, specially for $\rho \ll 1$. We have shown elsewhere [7] that, with spatial lattices, unconditional imitation is a rule which yields different results for some specific games depending on the synchronicity of the update, but to our knowledge this large sensitivity in the case of scale-free networks has not been reported in the literature and so it deserves further investigation.

In conclusion, we have introduced an evolutionary rule that allows to relax the demanding requirements of the unconditional imitation rule, while maintaining the basic properties of imitative behavior and local information. We have found that, for a wide range of $\rho$, the cooperation levels that it yields are not only preserved but in many cases they are even enhanced. This conclusion is general and independent of the details of the model; hence the reported mechanism might have an impact on many other evolutionary games. This work thus offers a new perspective on the significance of imitative dynamics, in general, and unconditional imitation, in particular. Instead of considering the promotion of cooperation ascribed to this rule as a singularity, we can now see it as 
a more robust outcome. Our results suggest that rather than the lack of stochasticity of unconditional imitation, it is the fast time scale what constitutes its key feature. Note that the evolution with unconditional imitation under asynchronous update is equivalent to some extent of stochastic perturbation to the synchronous case, and nonetheless the outcome is similar for most games on degree-homogeneous networks [7].

Finally, and from a more general viewpoint, this result belongs to a wider class of paradoxical behaviors originated in ratchet effects resulting from the combination of two dynamics, Parrondo's paradox being the best known example [21-23].

$$
* * *
$$

We thank G. SzABó for a critical reading of the manuscript. This work is supported by MICINN (Spain) under Grants Ingenio-MATHEMATICA and MOSAICO, and by Comunidad de Madrid (Spain) under Grants SIMUMAT-CM and MOSSNOHO-CM.

\section{REFERENCES}

[1] Pennisi E., Science, 309 (2005) 93.

[2] Gintis H., Game Theory Evolving (Princeton University Press) 2000.

[3] NowaK M. A., Science, 314 (2006) 1560.

[4] Szabó G. and Fáth G., Phys. Rep., 447 (2007) 97.

[5] Hofbauer J. and Sigmund K., Evolutionary Games and Population Dynamics (Cambridge University Press) 1998.

[6] NowaK M. A. and May R. M., Nature, 359 (1992) 826.

[7] Roca C. P., Cuesta J. A. and Sánchez A., arXiv: 0806.1649 [q-bio.PE] (2008).
[8] Roca C. P., Cuesta J. A. and Sánchez A., to be published in Phys. Life Rev. (2009) DOI: http://dx.doi.org/10.1016/j.plrev.2009.08.001.

[9] Pingle M. and Day R. H., J. Econ. Behav. Organ., 29 (1996) 191.

[10] Apesteguía J., Huck S. and Oechssler J., J. Econ. Theory, 136 (2006) 217.

[11] Helbing D., A mathematical model for behavioral changes through pair interactions, in Economic Evolution and Demographic Change, edited by HaGG G., Mueller U. and Troitzsch K. G. (Springer-Verlag, Berlin) 1992, pp. 330-348.

[12] Nowak M. A., Bonhoeffer S. and May R. M., Proc. Natl. Acad. Sci. U.S.A., 91 (1994) 4877.

[13] Santos F. C., Pacheco J. M. and Lenaerts T., Proc. Natl. Acad. Sci. U.S.A., 103 (2006) 3490.

[14] Licht A. N., Yale J. Int. Law, 24 (1999) 61.

[15] Axelrod R. and Hamilton W. D., Science, 211 (1981) 1390.

[16] Skyrms B., The Stag Hunt and the Evolution of Social Structure (Cambridge University Press) 2003.

[17] Maynard-Smith J. and Price G. R., Nature, 246 (1973) 15.

[18] Sugden R., Economics of Rights, Co-operation and Welfare, 2nd edition (Palgrave Macmillan, Hampshire) 2004.

[19] Szabó G., Vukov J. and Szolnoki A., Phys. Rev. E, 72 (2005) 047107.

[20] Perc M., New J. Phys., 8 (2006) 22.

[21] Harmer G. P. and Abвott D., Nature, 402 (1999) 864.

[22] Parrondo J. M. R., Harmer G. P. and Abbott D., Phys. Rev. Lett., 85 (2000) 5226.

[23] Parrondo J. M. R. and Dinis L., Contemp. Phys., 45 (2004) 147.

[24] Schweitzer F., Behera L. and Mühlenbein H., Adv. Complex Syst., 5 (2002) 269.

[25] Albert R. and Barabási A.-L., Rev. Mod. Phys., 74 (2002) 47. 